\title{
ARTICLE
}

Received 23 Nov 2015 | Accepted 5 Feb 2016 | Published 24 Mar $2016 \quad$ DOl: 10.1038/ncomms10959 OPEN

\section{Pterosin B prevents chondrocyte hypertrophy and osteoarthritis in mice by inhibiting Sik3}

Yasuhito Yahara ${ }^{1,2}$, Hiroshi Takemori ${ }^{3}$, Minoru Okada1, Azuma Kosai ${ }^{1}$, Akihiro Yamashita1, Tomohito Kobayashi', Kaori Fujita', Yumi Itoh ${ }^{3}$, Masahiro Nakamura ${ }^{4}$, Hiroyuki Fuchino ${ }^{5}$, Nobuo Kawahara ${ }^{5}$, Naoshi Fukui ${ }^{6}$, Akira Watanabe ${ }^{4}$, Tomoatsu Kimura ${ }^{2} \&$ Noriyuki Tsumaki ${ }^{1}$

Osteoarthritis is a common debilitating joint disorder. Risk factors for osteoarthritis include age, which is associated with thinning of articular cartilage. Here we generate chondrocytespecific salt-inducible kinase 3 (Sik3) conditional knockout mice that are resistant to osteoarthritis with thickened articular cartilage owing to a larger chondrocyte population. We also identify an edible Pteridium aquilinum compound, pterosin B, as a Sik3 pathway inhibitor. We show that either Sik3 deletion or intraarticular injection of mice with pterosin B inhibits chondrocyte hypertrophy and protects cartilage from osteoarthritis. Collectively, our results suggest Sik3 regulates the homeostasis of articular cartilage and is a target for the treatment of osteoarthritis, with pterosin B as a candidate therapeutic.

\footnotetext{
${ }^{1}$ Department of Cell Growth and Differentiation, Center for iPS Cell Research and Application, Kyoto University, 53 Kawahara-cho, Shogoin, Sakyo-ku, Kyoto 606-8507, Japan. ${ }^{2}$ Department of Orthopaedic Surgery, Faculty of Medicine, University of Toyama, 2630, Sugitani, Toyama 930-0194, Japan. ${ }^{3}$ Laboratory of Cell Signaling and Metabolic Disease, National Institutes of Biomedical Innovation, Health and Nutrition, 7-6-8, Asagi, Saito, Ibaraki, Osaka 567-0085, Japan. ${ }^{4}$ Genome/Epigenome Analysis Core Facility, Center for iPS Cell Research and Application, Kyoto University, 53 Kawahara-cho, Shogoin, Sakyo-ku, Kyoto 606-8507, Japan. ${ }^{5}$ Research Center for Medicinal Plant Resources, Tsukuba Division, National Institutes of Biomedical Innovation, Health and Nutrition, 1-2, Hachimandai, Tsukuba, Ibaraki 305-0843, Japan. ${ }^{6}$ Graduate School of Arts and Sciences, Department of Life Sciences, The University of Tokyo, Komaba 3-8-1, Meguro-ku, Tokyo 153-8902, Japan. Correspondence and requests for materials should be addressed to N.T.

(email: ntsumaki@cira.kyoto-u.ac.jp).
} 
A rticular cartilage lubricates the ends of bones. Its loss can ultimately result in osteoarthritis, a common debilitating joint disorder that affects more than $15 \%$ of the population over the age of 60 (refs 1,2). Common risk factors for osteoarthritis include age, excess mechanical loading and metabolic syndrome. The most apparent age-associated change is the reduced thickness of articular cartilage ${ }^{3,4}$; however, whether thinner cartilage is more susceptible to osteoarthritis is unclear. Although extensive studies have revealed age-related molecular changes in chondrocytes, the molecules that control thickness of articular cartilage are unknown.

During development, epiphyseal cartilage at both ends of the bone gives rise to articular cartilage and growth plate cartilage after the formation of the secondary ossification centre. Chondrocytes express type II collagen (COL2) and type XI collagen (COL11) during proliferation, but will ultimately stop proliferation and undergo hypertrophy in epiphyseal cartilage. Hypertrophic chondrocytes express type X collagen (COL10) and matrix metalloproteinases (MMPs), which contribute to the degradation of cartilage and replacement by bone. Articular cartilage consists of two layers, a non-calcified zone and a calcified zone, which are separated by the tidemark. Chondrocytes in the non-calcified zone express COL2 and COL11, whereas chondrocytes in the calcified zone express COL10. However, chondrocytes in the non-calcified zone express COL10 and MMPs in osteoarthritic conditions ${ }^{5-7}$, suggesting activation of the hypertrophy programme. Inhibiting these hypertrophic changes has been shown to reduce the severity of cartilage lesions in mouse osteoarthritis models ${ }^{6,8,9}$. Thus, molecules that activate the hypertrophy programme might be promising therapeutic targets for osteoarthritis drugs.

We previously reported that Sik3 knockout mice have delayed chondrocyte hypertrophy in mice ${ }^{10}$. Here we show that SIK3 is activated in human osteoarthritic cartilage, leading us to hypothesize that Sik3 is important for the development of osteoarthritis. However, Sik3 total knockout mice have severely malformed skeletons and most die at birth ${ }^{10}$; therefore, we generated tamoxifen conditional chondrocyte-specific Sik3 knockout mice. Deletion of Sik3 in adult mice causes thickening of articular cartilage and resistance to surgically induced osteoarthritis, a phenotype associated with reduced expression of Col10 in the non-calcified zone of articular cartilage. We screen for compounds that inhibit SIK3 activity, identifying pterosin B as a suppressor of Sik3 signalling. Intraarticular administration of pterosin B protects the mice from osteoarthritic change and Col10 expression. These results suggest that pterosin $\mathrm{B}$ is a promising candidate for osteoarthritis drug development.

\section{Results}

Expression of phospho-SIK3 in osteoarthritic cartilage. We investigated the expression of total SIK3 and phosphorylated SIK3 at T163 (pSIK3), which is the active form of SIK3 (refs 11,12), in articular cartilage. Immunohistochemical analysis revealed that total SIK3 was uniformly detected from the surface to deep layer, whereas pSik3 was detected only in the deep layer of normal articular cartilage (Supplementary Fig. 1a). On the other hand, pSIK3 was expressed in the superficial layer of severely affected osteoarthritic articular cartilage (Supplementary Fig. 1b). Moreover, the worse the OARSI grade ${ }^{13}$, the more cells in the superficial layers expressed pSIK3. These results suggest that activation of SIK3 is related to osteoarthritis development.

Generation of inducible Sik3 conditional knockout mice. We generated transgenic mice bearing the Col11a2-CreER construct, in which the CreER gene is linked to chondrocyte-specific $\alpha 2(\mathrm{XI})$ collagen chain gene (Col11a2) promoter/enhancer sequences (Supplementary Fig. 2a). We mated Col11a2-CreER transgenic mice with Rosa26-stop flox $-E Y F P$ tester mice to prepare Col11a2CreER; Rosa26-stop flox $-E Y F P$ mice. On intraperitoneal injection

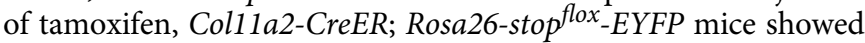
YFP fluorescence exclusively in primordial cartilage in the limbs and ribs of embryos at 14.5 days postcoitum, whereas Rosa26stop ${ }^{\text {flox }}$-EYFP mice showed no YFP fluorescence (Supplementary Fig. 2b). Col11a2-CreER; Rosa26-stop flox $_{\text {-EYFP mice treated with }}$ tamoxifen showed YFP expression exclusively in articular cartilage in knee joints at 16 weeks of age, whereas mice without tamoxifen treatment showed no YFP expression (Supplementary Fig. 2c). These results suggest that the Col11a2-CreER transgenic allele can induce recombination at $\operatorname{lox} P$ sites specifically in chondrocytes in a tamoxifen-dependent manner.

We next generated Sik3 $3^{\text {flox/ }}+$ (Supplementary Fig. 3a) mice and prepared Coll1a2-CreER; Sik3 flox/flox mice. Coll1a2CreER; Sik $3^{\text {flox } /+}$ mice and Sik $3^{\text {flox/flox }}$ mice were used as controls. We confirmed that Sik3 was deleted in the chondrocytes of Col11a2-CreER; Sik3 flox/flox mice after administration of tamoxifen (Supplementary Fig. 3b). Hereafter, we call tamoxifentreated Col11a2-CreER; Sik3 flox/flox mice as Sik3 ${ }^{\Delta / \Delta}$ mice, tamoxifen-treated Col11a2-CreER; Sik $3^{\text {flox } /+}$ mice as $S i k 3^{\Delta /+}$ mice and tamoxifen-treated $S i k 3^{\text {flox } / f l o x}$ mice as $S i k 3^{f / f}$ mice.

Deletion of Sik3 increases thickness of articular cartilage. To analyse Sik3 function in articular cartilage after birth, we treated mice with tamoxifen at 2 weeks of age and killed them at 4 weeks of age (Fig. 1a). To trace the fate of $S i k 3^{\Delta / \Delta}$ cells, we performed this experiment on the Rosa26-stop flox-EYFP background (Col11a2-CreER; Sik $3^{\text {flox/flox; }}$ Rosa26-stop flox $-E Y F P$ mice). Histological sections were stained with toluidine blue and von Kossa to identify total articular cartilage (non-calcified zone and calcified zone) and the non-calcified zone only. The thicknesses of total articular cartilage and the non-calcified zone of articular cartilage as well as that of growth plate cartilage were all increased in Sik3 ${ }^{\Delta / \Delta}$ mice compared with Sik3 ${ }^{\Delta /+}$ mice (Fig. 1b). In addition, the numbers of YFP-positive cells per surface in articular cartilage and growth plate cartilage in Sik3 $3^{\Delta / \Delta}$ mice were increased compared with those in $S i k 3^{\Delta{ }^{4}+}$ mice (Fig. 1c). The expression levels of Col2al and Acan did not differ between pellets of Col11a2-CreER; Sik $3^{\text {flox/flox }}$ chondrocytes cultured in the presence of tamoxifen and those in the absence of tamoxifen (Supplementary Fig. 3c), suggesting that the amount of matrix production per chondrocyte did not change. These results collectively suggest that Sik3 deletion increased the population of chondrocyte progenies, resulting in increased total amount of matrix and increased cartilage thickness.

We hypothesized that the increased chondrocyte population in $S i k 3^{\Delta / \Delta}$ mice might be caused either by increased proliferation rates or decreased removal rates of the cells. To analyse the proliferation rates, we performed BrdU labelling and found that Sik3 deletion had no effect on either articular or growth plate cartilage (Supplementary Fig. 4a). BrdU-positive cells were absent in articular cartilage in both $S i k 3^{\Delta / \Delta}$ mice and Sik3 $3^{\Delta /+}$ mice for the entire labelling period $(3 \mathrm{~h})$. On the other hand, immunohistochemical analysis showed that deletion of Sik3 almost eliminated Col10 expression in articular and growth plate cartilage (Fig. 1c), suggesting that the removal of chondrocytes was compromised. Lineage tracing revealed that progenies of Col11a2-marked YFP-positive chondrocytes expressed Col10 in the hypertrophic zone of growth plate cartilage (Fig. 1cA, arrow) and in the calcified zone of articular cartilage (Fig. 1cB, arrow) in control mice, but that Sik3-deficient chondrocytes did not express Col10 in either (Fig. $1 \mathrm{cC}$ and $1 \mathrm{cD}$ ). Moreover, YFP-positive cells were absent from the Col10-positive area in $S i k 3^{\Delta / \Delta}$ mice 


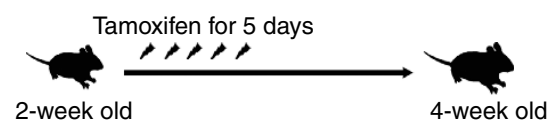

b
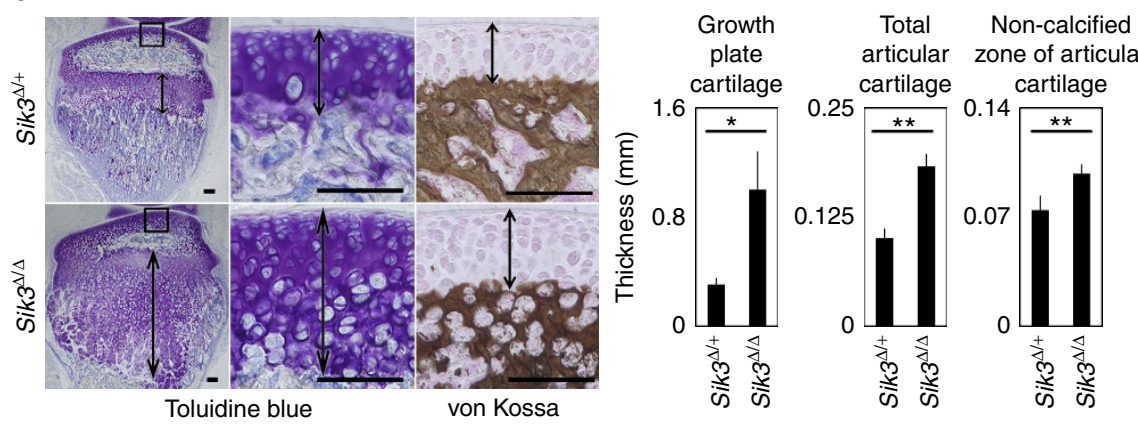

C
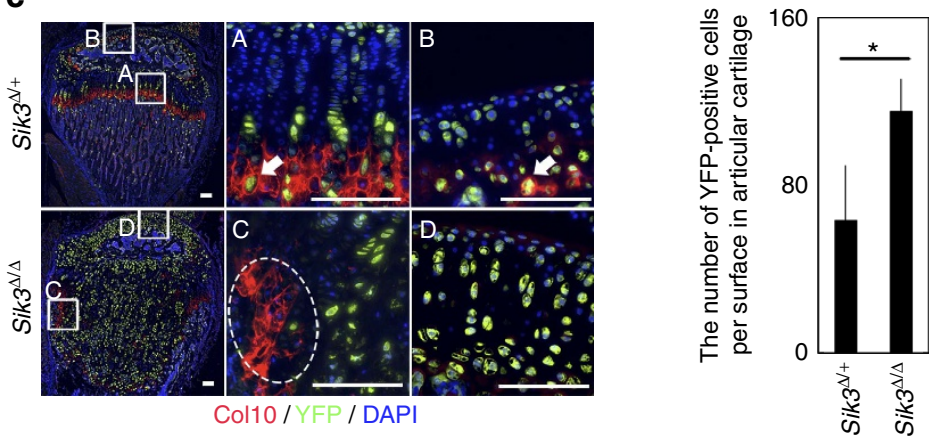

d

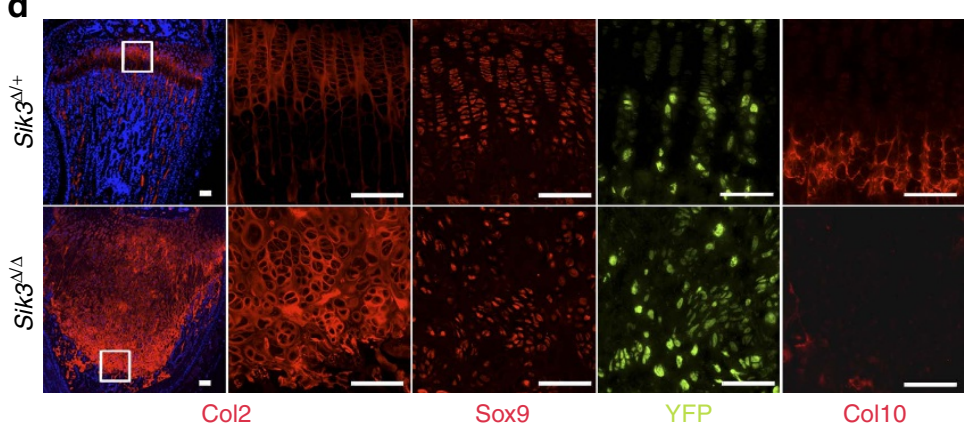

Figure 1 | Phenotype of cartilage in mice lacking Sik3 after birth. (a) We injected Col11a2-CreER; Sik3flox/flox; Rosa26-stopflox-EYFP mice and Col11a2-CreER; Sik $3^{f l o x} /+$; Rosa26-stop flox -EYFP mice with tamoxifen daily for 5 consecutive days beginning at 2 weeks of age and killed them at 4 weeks of age.

(b) Semiserial histological sagittal sections of proximal tibia stained with toluidine blue and von Kossa. Boxed regions are shown to the right. Thickness of growth plate cartilage, total articular cartilage and non-calcified articular cartilage at the vertex of the articular surface in the corresponding sections of the weight-bearing area (indicated by arrows) were measured and are shown in the right graphs. $n=4$ mice. (c) Semiserial sections of $\mathbf{b}$ were immunostained for Col10 (red) and YFP (yellow). Blue colour is DAPI. Boxed regions indicated by A-D are shown to the right. The numbers of EYFP-positive cells per $360 \mu \mathrm{m}$-surface in articular cartilage were counted and are shown in the right graph. Arrows, Col11a2-marked YFP-positive chondrocytes in Col10-positive matrix in Sik $3^{\Delta /+}$ mice. Dotted circle, the Col10-positive area lacking YFP-positive cells in Sik3 $3^{\Delta / \Delta}$ mice; $n=4$ mice. (d) Semiserial sections of b,c were immunostained for Col2, Sox9, YFP and Col10. Boxed regions in the left panels (Col2) are shown in the right panels. The images are representative of two independent experiments. Error bars denote means \pm s.d. ${ }^{\star} P<0.05$ and ${ }^{\star \star} P<0.01$ by the $t$-test. Scale bars, $100 \mu \mathrm{m}$.

(Fig. 1cC, dotted circle), suggesting that progenies of $\mathrm{Sik}^{\mathrm{A} / 4}$ chondrocytes continued to express $\mathrm{Col} 2$ instead. We confirmed that Sik3-deficient cells expressed Col2 and Sox9, but not Col10 in growth plate cartilage (Fig. 1d, bottom panels), indicating that Sik3-deficient cells remain chondrocytes. These results collectively suggest that Sik3 deletion keeps the Col2-positive; Col10-negative chondrocyte state, which increases the population of Col2-positive; Col10-negative chondrocytes and thickening of articular cartilage.
Sik3 deletion protects cartilage from osteoarthritis. We next examined whether Sik3 deletion affected the development of osteoarthritis in adult mice. We treated 7-week-old mice with tamoxifen to prepare $S i k 3^{\Delta / \Delta}$ and Sik3/ff mice, and subsequently subjected their right knees to destabilized medial meniscus (DMM) surgery or sham surgery (skin and joint capsule incision) at 8 weeks of age. The mice were killed 8 weeks after the operation (therefore at 16 weeks of age) and subjected to histological analysis (Fig. 2a). The thickness of articular cartilage in 
sham-operated knees in $S i k 3^{\Delta / \Delta}$ mice was thicker than that in $S i k 3^{f / f}$ mice (Fig. 2b), confirming that Sik3 deletion caused thickening of articular cartilage in adult mice. DMM operation caused less severe osteoarthritis in $S i k 3^{\Delta / \Delta}$ mice than in $S i k 3^{f / f}$ mice (Fig. 2c). Immunohistochemical analysis showed that DMM operation induced Col10 expression in the non-calcified zone of articular cartilage of $S i k 3^{f / f}$ mice (Fig. 2d). Deletion of Sik3 reduced this induction, as indicated by the limited expression of Collo in the $S i k 3^{\Delta / \Delta}$ mouse knee joint that received the DMM operation. These results collectively suggest that deletion of Sik3 in chondrocytes thickens articular cartilage and inhibits activation of the hypertrophy programme to protect mice from developing osteoarthritis.

We further examined whether Sik3 deletion in chondrocytes affects extra-cartilage status, which could affect cartilage biology and pathology. Body weights and locomotor activities indicated by a rotarod performance test were not different between Sik3 $3^{\text {flox/flox }}$ and Col11a2-CreER; Sik3 flox/flox mice before or 8 weeks after administration of tamoxifen (Supplementary Fig. 4b). Angiogenesis indicated by the amount of CD31-positive endothelial progenitor cells in subchondral bone was also not different between Sik3 flox/flox and Col11a2-CreER; Sik3 $3^{\text {flox/flox }}$ mice after administration of tamoxifen (Supplementary Fig. 4c). These results suggest that $S i k 3$ deletion in cartilage affected cartilage biology and pathology autonomously.

Identification of pterosin $B$ as a Sik3 inhibitor. Because our $S i k 3^{\Delta / \Delta}$ mice data suggested that Sik3 could be a target for treatment of osteoarthritis, we searched for compounds that inhibit Sik3 activity. It is known that SIKs phosphorylate HDACs and CREB-regulated transcription coactivators (CRTCs) to translocate these molecules from the nucleus to cytoplasm, which thus abolishes their function in the nucleus. HDAC inhibits myocyte enhancer factor 2C (MEF2C), and CRTC activates CREB in nuclei. We established a dual luciferase assay system to monitor MEF2C and CRTC2 activities in HEK293 cells. We screened $\sim 2,500$ compounds and found that pterosin B (Fig. 3a) decreased MEF2C activity and increased CRTC2 activity ${ }^{14}$. Pterosin B is an extract acquired from boiling Pteridium aquilinum in water supplemented with sodium bicarbonate. We then developed a method to synthesize pterosin B (Supplementary Fig. 5a). Our synthesized pterosin B inhibited MEF2C and activated CRTC2 in a dose-dependent manner in HEK293 cells according to a luciferase assay system in which luciferase activity was regulated by GAL4-fusion MEF2 or GAL4-fusion CRTC2 (Supplementary Fig. 5b). These results confirmed that pterosin $\mathrm{B}$ and not some impurity in the extract was responsible for the function. Further, we confirmed that pterosin B specifically inhibited Sik3 in ATDC5 cells (Supplementary Fig. 5c). Thus, we used synthesized pterosin B in the following experiments.

Western blot analysis showed that the addition of pterosin $\mathrm{B}$ decreased the amounts of Sik3, phosphorylated Hdac4 and phosphorylated $\mathrm{Crtc1}$ in primary chondrocytes in a manner similar to 4-OH tamoxifen in chondrocytes from Col11a2-CreER; Sik $3^{\text {flox/flox }}$ mice (Fig. 3b).

Sik3 accelerates chondrocyte hypertrophy by anchoring Hdac4 to the cytoplasm and releasing Mef2c from inhibition by Hdac4 in the nucleus ${ }^{10}$. Mef2c accelerates the hypertrophy programme in chondrocytes ${ }^{15}$. We examined whether pterosin B inhibits hypertrophic differentiation of chondrocytes by performing pellet culture of wild-type mouse primary chondrocytes in the presence or absence of pterosin B for 4 weeks. The presence of pterosin B decreased the expression levels of Col10, Mef2c and Alp in a dosedependent manner (Fig. 3c), similar to how tamoxifen decreased a

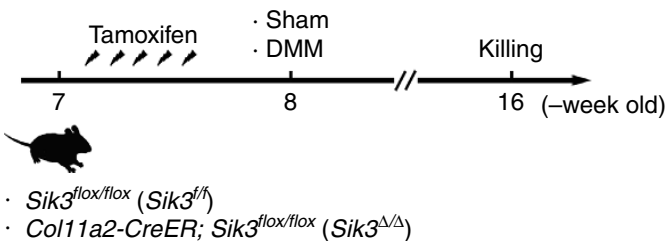

b
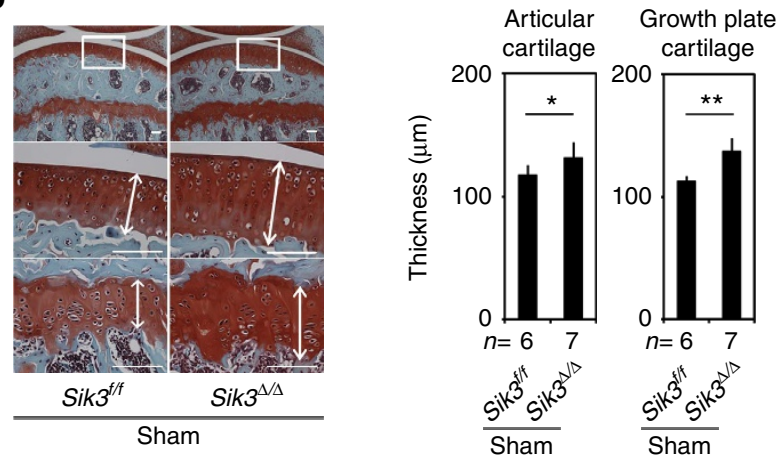

C
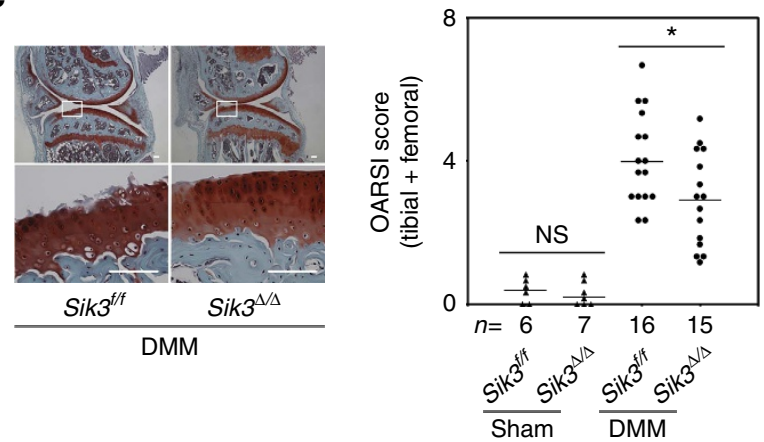

d
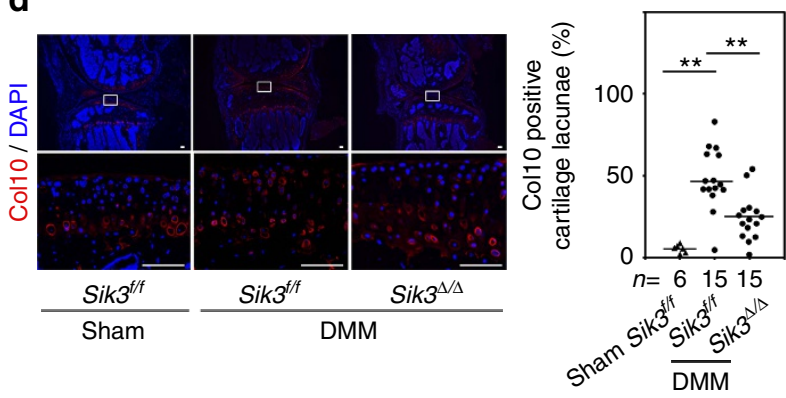

Figure 2 | Effects of Sik3 deletion on osteoarthritis development in DMM-operated knees. (a) Protocol of experiments: 7-week-old mice were intraperitoneally injected with tamoxifen for 5 consecutive days, had their right knees subjected to DMM or sham surgeries, and were killed at 16 weeks of age. (b) Histology of proximal tibia in sham-operated knees. Safranin O-fast green-iron haematoxylin staining. The thickness of articular cartilage and growth plate cartilage are shown in the graph. $t$-test.

(c) Histology of articular cartilage of proximal tibia in DMM-operated knees. Boxed regions are shown in the bottom panels. OARSI scores of articular cartilage in sham- and DMM-operated knees are shown. Two blinded scorers gave consistent results. Mann-Whitney test. (d) Immunohistochemistry for Col10 in knees. Boxed regions in the top panels are shown in the bottom panels. The percentage of lacunae surrounded by Col10-positive matrix per total number of lacunae are shown in the right graph. Tukey-Kramer post hoc test. Error bars denote means \pm s.d. Numbers of mice examined are indicated at the bottom of the graphs. ${ }^{\star} P<0.05$ and ${ }^{\star \star} P<0.01$. NS, not significantly different. Scale bars, $100 \mu \mathrm{m}$. 
a<smiles>CC(=O)c1cc2c(c(C)c1CCO)C(=O)C(C)C2</smiles>

b

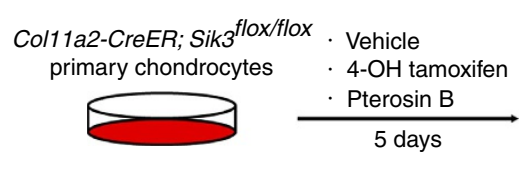

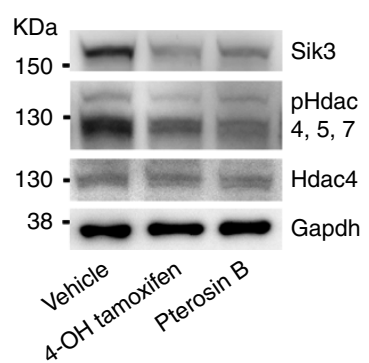

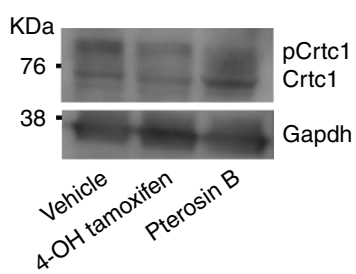

C
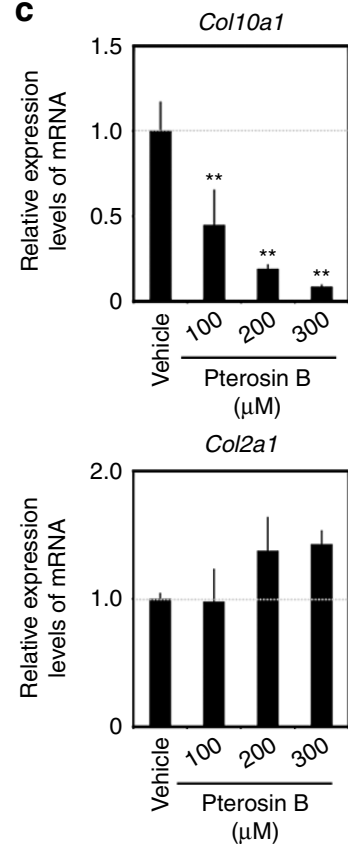
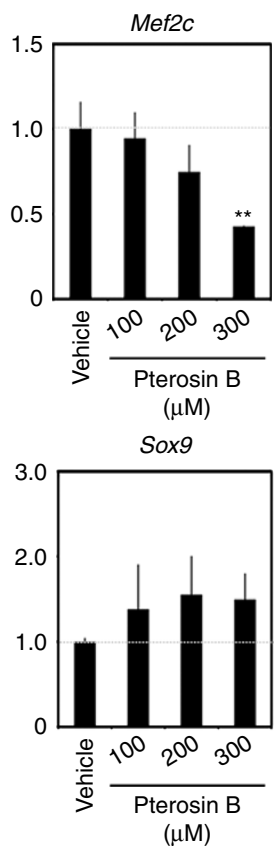
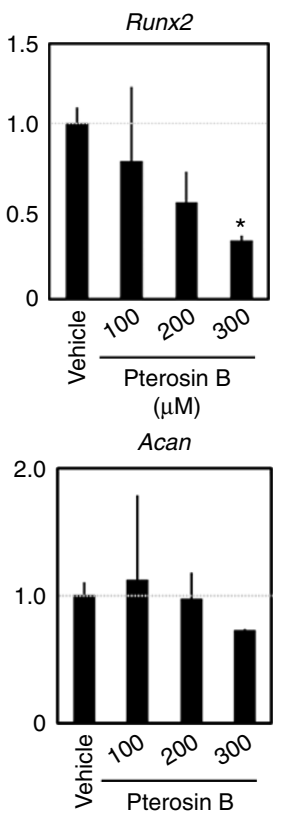

$(\mu \mathrm{M})$
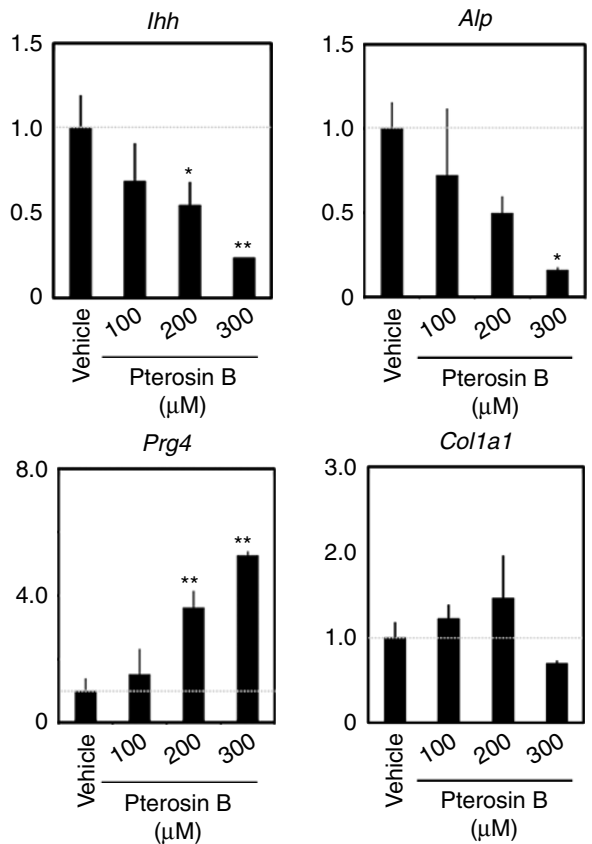

d
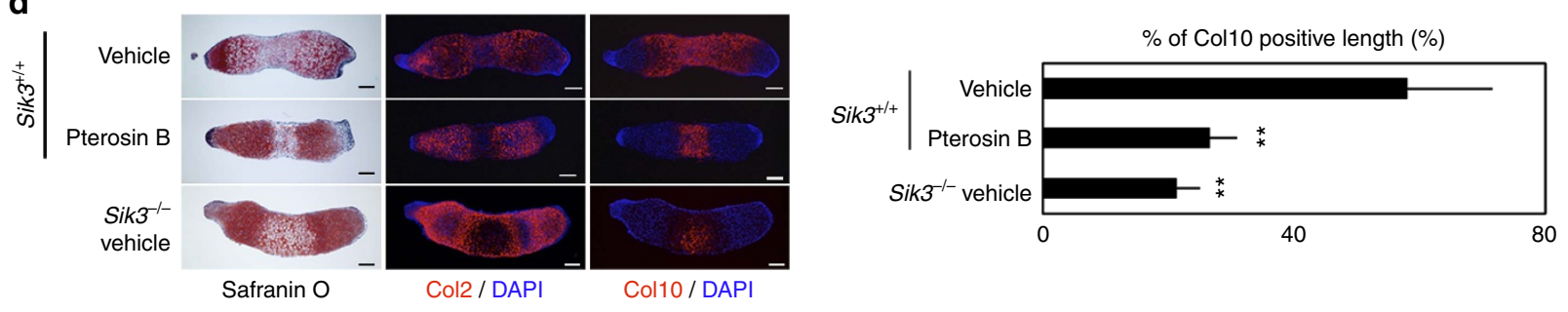

e

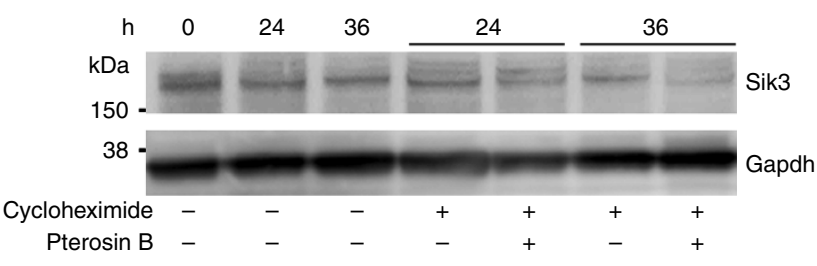

f

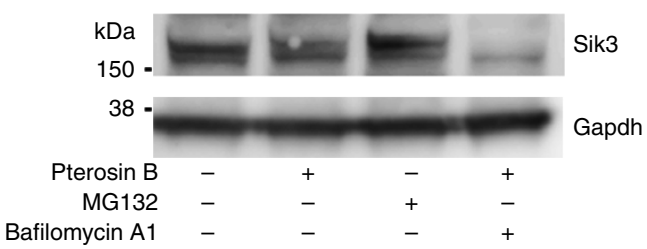

Figure 3 | Effects of pterosin B on chondrocyte differentiation in vitro. (a) Structure of pterosin B. (b) Primary chondrocytes prepared from Col17a2-CreER; Sik $3^{\text {flox/flox }}$ mice were treated with vehicle, $0.5 \mu \mathrm{M} 4-\mathrm{OH}$ tamoxifen or $300 \mu \mathrm{M}$ pterosin $\mathrm{B}$ for 5 days and subjected to western blot analysis. The images are representative of two independent experiments. (c) Real-time RT-PCR expression analysis of marker genes in pellet culture of mouse primary chondrocytes with pterosin $\mathrm{B} ; n=3$ pellets. (d) Metatarsal primordial cartilage from Sik3 ${ }^{+/+}$mice were organ-cultured in $300 \mu \mathrm{M}$ pterosin B or vehicle. Metatarsal primordial cartilage from conventional Sik3 knockout mice were used for the control. Semiserial histological sections were stained with safranin O-fast green-iron haematoxylin and immunostained for Col2 and Col10. The percentage of Col10-postive area per total length in the axial direction is indicated; $n=3$ metatarsals. (e) Primary chondrocytes were treated with $100 \mu \mathrm{g} \mathrm{ml} \mathrm{m}^{-1}$ cycloheximide in the presence or absence of $300 \mu \mathrm{M}$ pterosin B. Cells were lysed after treatment for 0, 24 and $36 \mathrm{~h}$, and cell lysates were subjected to western blot analysis for Sik3 and Gapdh. The images are representative of two independent experiments. (f) Primary chondrocytes were incubated in the presence or absence of $1 \mu \mathrm{M} \mathrm{MG} 132,10 \mathrm{nM}$ bafilomycin $\mathrm{A} 1$ or $300 \mu \mathrm{M}$ pterosin B for $36 \mathrm{~h}$. Cell lysates were subjected to western blot analysis for Sik3 and Gapdh. The images are representative of two independent experiments. Error bars denote the means \pm s.d. ${ }^{\star} P<0.05$ and ${ }^{*} P<0.01$ by the Tukey-Kramer post hoc test. Scale bars, $100 \mu \mathrm{m}$. 
these levels in pellet culture of Col11a2-CreER; Sik3 flox/flox chondrocytes (Supplementary Fig. 3c). It also significantly decreased Runx2 and Ihh expression levels (Fig. 3c), suggesting that pterosin B inhibits prehypertrophy of chondrocytes as well. On the other hand, we did not detect significant changes in the expression levels of Sox9, Col2a1 or Acan mRNAs in pellets with pterosin B treatment (Fig. 3c), which resembles tamoxifen effects in pellet culture of Col11a2-CreER; Sik $3^{\text {flox/flox }}$ chondrocytes (Supplementary Fig. 3c). We suspect the expression levels of these three factors did not increase when hypertrophy was inhibited by Sik3 deletion or pterosin $B$, because the pellet culture samples were mainly composed of Col2a1-expressing chondrocytes and contained a limited population of hypertrophic chondrocytes. Finally, pterosin B did not significantly affect the expression of catabolic enzymes, such as Mmp3, Mmp13, Adamts4 or Adamts5 (Supplementary Fig. 6a).

In addition, pterosin B increased the expression level of Prg4 (Fig. 3c), which encodes lubricin, in wild-type chondrocyte pellets. A CBP-CREB interaction inhibitor partly abolished this elevation of Prg4 expression, suggesting that pterosin B treatment activates Prg4 expression, at least in part, through the CRTC/ CREB pathway (Supplementary Fig. 6b). We also confirmed that pterosin B inhibits chondrocyte hypertrophy in organ culture of metatarsal primordial cartilage (Fig. 3d), as it reduced the area of the Col10-positive region in organ-cultured primordial cartilage of wild-type mice.

RNA-sequencing analysis of entire mRNA transcripts showed that chondrocyte pellets cultured in the presence or absence of pterosin B and cartilage tissues were clustered distinctly from other tissues such as skin, bone, muscle, liver, kidney, small intestine, testis, brain and spinal cord (Supplementary Fig. 7). Real-time PCR with reverse transcription (RT-PCR) expression analysis showed pterosin $\mathrm{B}$ did not significantly change Colla1 expression levels (Fig. 3c). These results suggest that pterosin B regulates the differentiation of chondrocytes, but does not alter the cellular phenotype to other types of cells. RNA sequencing analysis (Supplementary Fig. 8) largely confirmed the changes in the expression of marker genes found by real-time RT-PCR analysis (Fig. 3c). Gene Ontology term analysis demonstrated that downregulated genes in pterosin B-treated cells were associated with bone mineralization (Supplementary Table 1), agreeing with the observation that pterosin B delays chondrocyte hypertrophy and mineralization. In addition, KEGG (Kyoto Encyclopedia of Genes and Genomes) pathway analysis indicated that hedgehog signalling was attenuated by pterosin B. The inhibition of hedgehog signalling attenuates the severity of osteoarthritis ${ }^{6}$. Whether hedgehog signalling mediates effects of Sik3 on osteoarthritis is unexplored.

We finally analysed how pterosin B affects SIK3 activity. Western blot analysis showed that treatment with pterosin B decreased the amount of Sik3 protein (Fig. 3b). This decrease was accelerated in cells treated with cycloheximide, an inhibitor of protein synthesis (Fig. 3e). We also found that MG132 increased the amount of Sik3 protein, whereas bafilomycin A1 did not (Fig. 3f). Combined, these results suggest that pterosin B induces the degradation of Sik3 protein by a proteasomal pathway. SIK3 is activated by phosphorylation at T163 (ref. 16) and inactivated by phosphorylation at T411 or S493 (ref. 17). We recently found that pterosin B upregulates the phosphorylation of SIK3 at T411 and S493 in hepatocytes, which results in the suppression of SIK3 activity $^{14}$. Whether phosphorylation at T411 or S493 is related to SIK3 degradation in chondrocytes is for further study.

Pterosin B protects cartilage from osteoarthritis. We examined whether pterosin B can control osteoarthritis development. We performed DMM surgery on the right knees of 8 -week-old wild-type mice (Fig. 4a). We injected pterosin B or vehicle solutions into the joint space of DMM-operated knees three times a week for 8 weeks. We also injected the right knees of mice, which were not subjected to operation with pterosin B or vehicle using the same schedule. We killed the mice at 16 weeks of age. OARSI scores were better in the pterosin B-treated knees with DMM than vehicle-treated knees with DMM. The non-operated knees treated with pterosin B looked normal and showed similar OARSI scores with those of non-operated knees treated with vehicle. These results suggest that pterosin B protects mice from osteoarthritis development. Immunohistochemical analysis showed that pterosin B treatment reduced the Col10 expression activated by DMM in the non-calcified zone of articular cartilage (Fig. 4b). Pterosin B treatment did not significantly change the synovitis score in non-operated knees or DMM-operated knees (Fig. 4c).

We also examined the effects of pterosin $\mathrm{B}$ on older mice. We performed DMM surgery at 13 weeks of age and injected pterosin $\mathrm{B}$ or vehicle solutions into the joint space of the DMM-operated knees three times a week for 12 weeks (Fig. 4d). Again, OARSI scores were better in the pterosin B-treated knees with DMM than vehicle-treated knees with DMM (Fig. 4d). Pterosin B treatment reduced Col10 expression in the non-calcified zone of articular cartilage DMM-operated knees (Fig. 4e).

Pterosin B inhibits hypertrophy of human chondrocytes. We finally examined whether pterosin B inhibits hypertrophy of human articular chondrocytes. Human articular chondrocytes were subjected to pellets cultured in chondrogenic medium for 8 weeks. We further cultured the chondrocyte pellets in medium containing BMP-2, GDF-5, 3,3,5-Triiodo-L-thyronine (T3) and $\beta$-glycerophosphate for 2 weeks to induce hypertrophic differentiation. Histological analysis of the pellets revealed that the addition of pterosin $\mathrm{B}$ in the medium protected chondrocytes from hypertrophy and mineralization (Fig. 5a). Real-time RT-PCR analysis showed that pterosin $\mathrm{B}$ reduced the expression levels of COL10A1 and ALP, did not change that of COL $2 A 1$ and increased those of SOX9 and PRG4 (Fig. 5b).

We next examined the effects of pterosin B on chondrocytes derived from human-induced pluripotent stem cells (hiPSCs). We differentiated hiPSCs towards chondroyctes and subjected them to suspension culture to produce cartilage particles for 8 weeks, as we described previously ${ }^{18}$. The cartilage particles were further cultured with pterosin B or vehicle in hypertrophic condition for 4 weeks. Pterosin B protected chondrocytes in the particles from hypertrophy and mineralization, as indicated by histological appearance, von Kossa staining, immunohistochemical staining for COL10 (Fig. 5c) and the expression of marker genes (Fig. 5d). These results collectively suggest that pterosin B inhibits hypertrophic change of human articular chondrocytes and hiPSC-derived chondrocytes.

\section{Discussion}

Here we show that genetic deletion of Sik3 or inhibition of the Sik3 signal by pterosin B protected mouse cartilage from osteoarthritic change caused by DMM. The mechanisms of the protection appear as inhibition of activation of the hypertrophy programme or elevation of the Prg4 expression level in articular chondrocytes.

Although definite evidence is lacking, age-associated thinning of articular cartilage is a suspect risk factor for the erosion of articular cartilage in osteoarthritis ${ }^{3}$. The turnover of articular cartilage, which should define the thickness of articular cartilage, is not well understood. The developmental process of the secondary ossification centre raises the hypothesis that cells in 
a
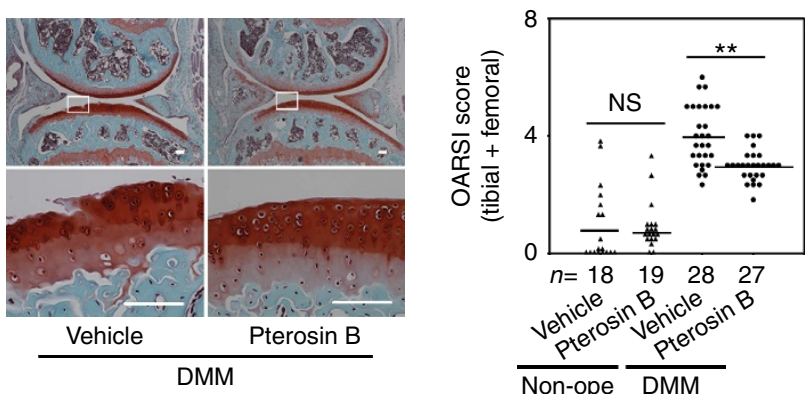

b

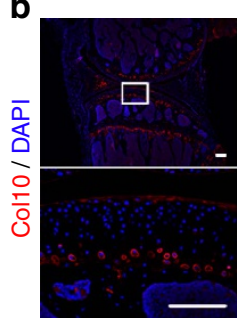

Vehicle
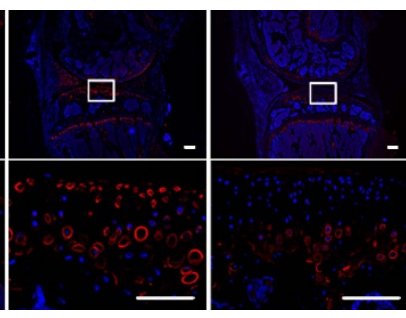

Non-ope

Vehicle

Pterosin B

C

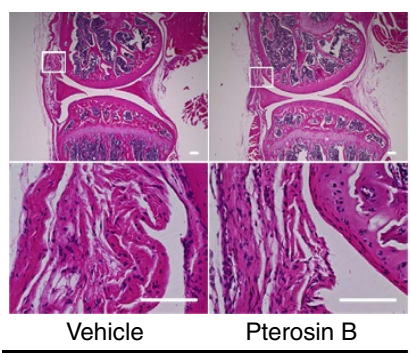

Non-ope

d
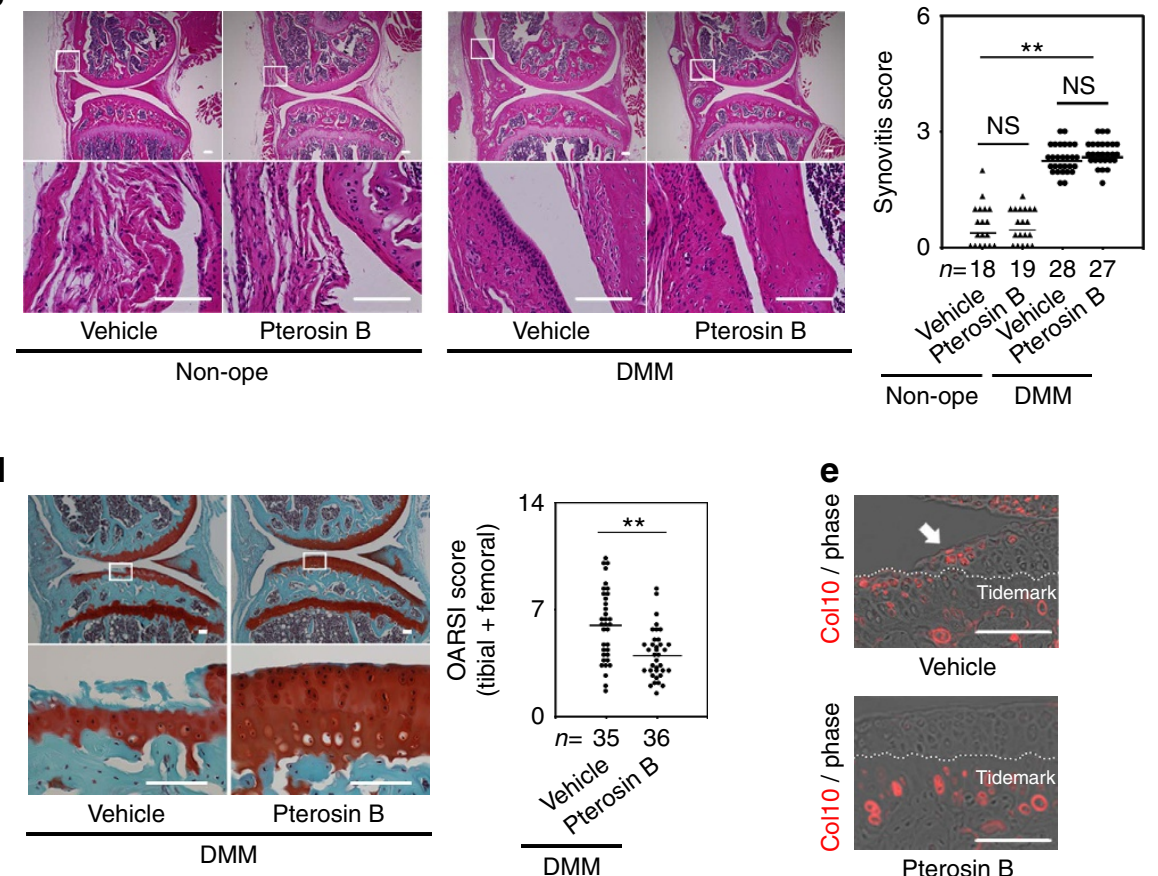

Figure 4 | Effects of pterosin B on osteoarthritis development in DMM-operated knees. (a-c) Eight-week-old mice with DMM were treated with pterosin B for 8 weeks. (a) Histology of articular cartilage of DMM-operated knees injected with pterosin B or vehicle. Safranin O-fast green-iron haematoxylin staining. Boxed regions in the top panels are shown in the bottom panels. OARSI scores of articular cartilage in DMM-operated knees injected with pterosin B or vehicle (black dots) are shown to the right. OARSI scoring was also done on non-operated knees injected with pterosin B or vehicle (black triangles). Two blinded scorers gave consistent results. Representative data are shown; Mann-Whitney test. (b) Immunohistochemistry for Col10 in knees. Boxed regions in the top panels are shown in the bottom panels. The percentage of lacunae surrounded by Col10-positive matrix per total number of lacunae is indicated. Tukey-Kramer post hoc test. (c) Histology of synovium; haematoxylin-eosin staining. Boxed regions in the top panels are shown in the bottom panels. Synovitis scores in DMM-operated knees injected with pterosin B or vehicle (black dots) and in non-operated knees injected with pterosin B or vehicle (black triangles); Mann-Whitney test. (d,e) Thirteen-week-old mice with DMM were treated with pterosin B for 12 weeks.

(d) Histology of articular cartilage of DMM-operated knees injected with pterosin B or vehicle. Safranin O-fast green-iron haematoxylin staining. Boxed regions in the top panels are shown in the bottom panels. OARSI scores of articular cartilage in DMM-operated knees injected with pterosin B or vehicle are shown to the right. Two blinded scorers gave consistent results. Representative data are shown; Mann-Whitney test. (e) Immunohistochemistry for Col10 in articular cartilage in DMM-operated knees injected with pterosin B or vehicle. White dotted lines indicate tidemarks. White arrow represents Col10 expression above the tidemark. The images are representative of two independent experiments. Number of mice examined is indicated at the bottom of the graphs. ${ }^{\star} P<0.05$ and ${ }^{\star} * P<0.01$. NS, not significantly different. Scale bars, $100 \mu \mathrm{m}$. 

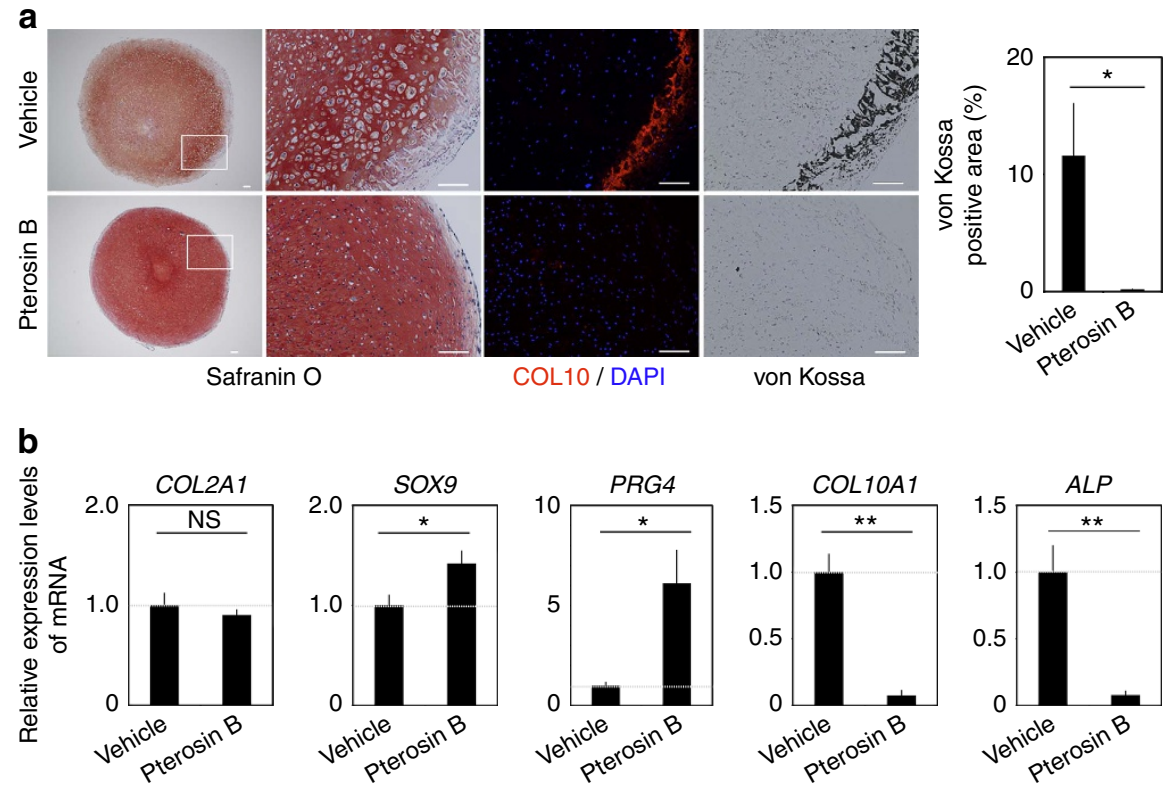

C
$\frac{0}{0}$
$\frac{0}{1}$
$\frac{10}{5}$
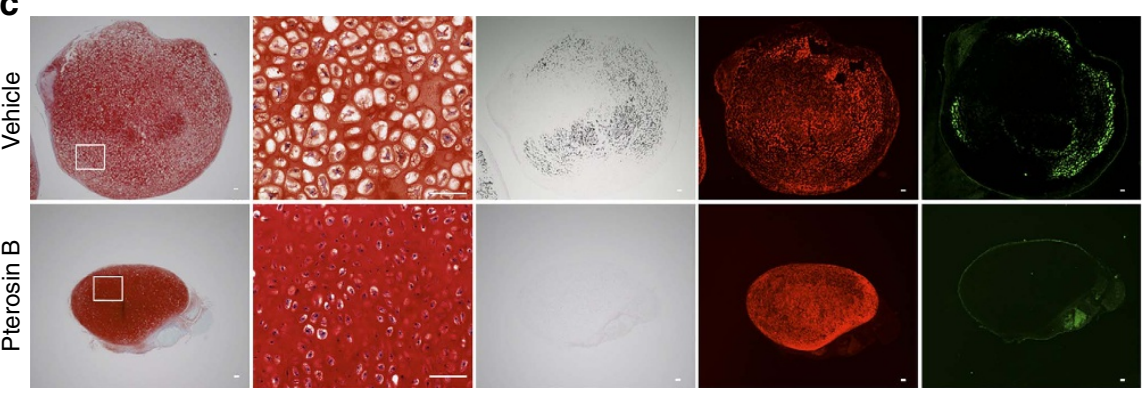

Safranin O

von Kossa

COL2
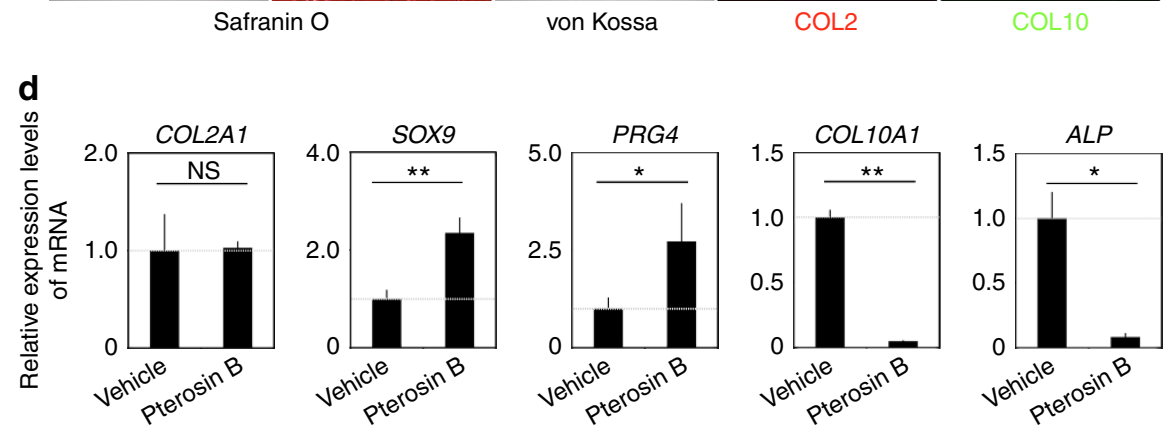

Figure 5 | Effects of pterosin B on human articular chondrocytes and hiPSC-derived chondrocytes. (a) Semiserial histological sections of cultured pellets of human articular cartilage in the presence or absence of $300 \mu \mathrm{M}$ pterosin B were stained with safranin O-fast green-iron haematoxylin and von Kossa and immunostained for COL10. Boxed regions in the left panels are shown in the right. Area of von Kossa-positive regions per total area of pellets are shown; $n=3$ pellets. (b) Real-time RT-PCR expression analysis of marker genes in pellet culture of human articular chondrocytes in the presence or absence of $300 \mu \mathrm{M}$ pterosin $\mathrm{B} ; n=3$ pellets. (c) Semiserial histological sections of hiPSC-derived cartilage particles treated with or without $300 \mu \mathrm{M}$ pterosin B were stained with safranin O-fast green-iron haematoxylin and von Kossa and immunostained for COL2 and COL10. Boxed regions in the left panels are shown in the right. The images are representative of three pellets each. (d) Real-time RT-PCR expression analysis of marker genes in hiPSC-derived cartilage particles in the presence or absence of $300 \mu \mathrm{M}$ pterosin $\mathrm{B} ; n=3$ pellets. Error bars denote means \pm s.d. ${ }^{\star} P<0.05$, ${ }^{\star *} P<0.01$ and NS, not significantly different by the $t$-test. Scale bars, $100 \mu \mathrm{m}$.

the non-calcified zone very slowly turn into cells that express Col10 and reside in the calcified zone, where they are subsequently removed and replaced by subchondral bone. A recent study using lineage tracing of Prg4-positive cells has shown that cells in the deeper zone are the progeny of cells in the non-calcified zone ${ }^{19}$, supporting this hypothesis. The thickness of articular cartilage decreases with age, probably due to an increasing imbalance between the proliferation rate of noncalcified zone cells and the rate by which these cells turn into cells that express Col10, reside in the calcified zone and are finally removed. In this study, we found that Sik3 deletion increased the population of chondrocytes and thickened articular cartilage at least in young mice. Examining the effects of Sik3 deletion on age-associated osteoarthritis model in mice is for further study.

Independent research groups have confirmed two types of substrates for SIKs, CRTC (a coactivator for the cAMP response element (CRE)-binding protein (CREB)] and class IIa histone deacetylase $(\mathrm{HDAC})^{20}$. SIKs phosphorylate these two substrates to induce nuclear export, resulting in a loss of the substrates' transcriptional regulatory activities ${ }^{21,22}$. Sik3 anchors Hdac4 in 
the cytoplasm to release Mef2c from inhibition by Hdac4, thus activating the hypertrophy programme in the nuclei of growth plate cartilage $\mathrm{c}^{10,15,23}$. It has been reported that HDAC4 is decreased in osteoarthritis cartilage, which may contribute to the pathogenesis of osteoarthritis cartilage degeneration ${ }^{24}$. These findings are consistent with our observation that Sik3 deletion or pterosin $\mathrm{B}$ administration protected cartilage from osteoarthritic change through activation of $\mathrm{Hdac} 4$, which prevented Mef2c from activating the hypertrophy programme in articular chondrocytes.

Prg4 is secreted by the most superficial articular chondrocytes and contributes to the lubrication of joints ${ }^{25}$. It also protects cartilage against osteoarthritic change ${ }^{26,27}$, and its expression is activated by the CRTC/CREB complex in articular chondrocytes $^{28}$. Sik3 anchors CRTC2 in the cytoplasm, preventing CRTC2 from co-activating CREB in nuclei ${ }^{29}$. We found that pterosin B treatment increased the expression level of Prg4 in chondrocytes, at least in part, through CREB activation. These findings collectively suggest that Sik3 deletion or pterosin B administration protect cartilage from osteoarthritic change through the CRTC/CREB pathway to activate Prg4 expression.

There are some limitations in this study. First, we cannot exclude the possibility that thickening of growth plates affected the course of osteoarthritis development in Sik $3^{\Delta / \Delta}$ mice. Second, DMM surgery was performed on relatively young $S i k 3^{\Delta / \Delta}$ mice. An analysis of the effects of Sik3 deletion on aged mice with DMM surgery would further clarify whether Sik3 affects the development of osteoarthritis, since osteoarthritis is more common in the elderly. Third, because we are not sure whether injections of pterosin B were always done intraarticularly and because pterosin $\mathrm{B}$ should be absorbed in the joint, we cannot dismiss extraarticular or systemic effects. Fourth and finally, other effects of pterosin B besides Sik3 inhibition cannot be ruled out as providing protection from osteoarthritis. Nevertheless, our study shows that SIK3 was activated in human osteoarthritic chondrocytes and that pterosin $\mathrm{B}$ inhibited hypertrophic changes and increased PRG4 expression in human articular chondrocytes and hiPSC-derived chondrocytes. Pterosin B is a compound in a cooked plant consumed in human diets. On the basis of our results, we suggest that SIK3 regulates the homeostasis of articular cartilage and can be a target for the treatment of osteoarthritis, and that pterosin B can be the lead compound for relevant drugs.

\section{Methods}

Ethics statement. All the experiments were approved by the institutional review board, institutional animal committee (as appropriate) and the institutional biosafety committee of Kyoto University, and the institutional review board of the University of Toyama and Hachiya Orthopaedic Hospital.

Generation of mice. A cDNA encoding the modified estrogen receptor ligand binding domain (ER) (provided by Dr M. Iwamoto, Children's Hospital of Philadelphia) $)^{30}$ was linked to the Cre sequence to create the CreER gene. The lacZ gene in 742LacZInt ${ }^{31}$ was replaced with the CreER gene to create Col11a2-CreER, and transgenic mice bearing the Col11a2-CreER were generated.

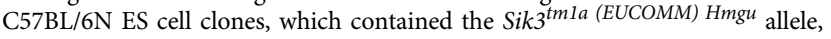
were purchased from the European Conditional Mouse Mutagenesis Program (EUCOMM). This allele includes targeting cassettes that were flanked by FRT recombination sites to allow removal by Flp recombinase and a pair of loxP recombination sites around exon 5 of Sik3 (Supplementary Fig. 3a). These targeted ES cells were injected into blastocysts of Slc:ICR mice to generate ES cell-derived chimeras. Male chimeras were mated with C57BL/6NCrSlc female mice, and germ line transmission was assessed by PCR. Heterozygous F1 mice were mated with B6$\mathrm{Tg}$ (CAG-FLPe) 36 mice, which were provided by the RIKEN BRC through the National Bio-Resource Project of MEXT, Japan, to delete the Neo and LacZ cassettes and generate a floxed allele $\left(\mathrm{Sik}^{f} f^{f o x /+}\right)$. Cre recombinases can induce gene inactivation of the Sik $3^{f l o x}$ allele by deleting exon 5 to generate a frame shift.

To generate Sik3 conditional knockout mice, Col11a2-CreER transgenic mice and Sik $3^{\text {flox/flox }}$ mice were mated. Then, Col11a2-CreER; Sik $3^{\text {flox/flox }}$ and Rosa26stop $^{\text {flox }}$-EYFP mice (Jackson Laboratory) were intercrossed and Col11a2-CreER;
Sik $3^{\text {flox/flox; }}$ Rosa26-stop ${ }^{\text {flox }}$-EYFP mice were generated. Col11a2-CreER; Sik $3^{\text {flox/+ }}$; Rosa26-stop ${ }^{\text {flox }}$-EYFP mice were used as controls.

Human articular cartilage samples and OARSI grading. Articular cartilages were collected from 16 patients suffering from medial type knee osteoarthritis at the time of total knee arthroplasty. A cartilage fragment was dissected from each individual and subjected to histological analysis. Normal articular cartilage samples were prepared from cadavers who had been free from joint disorders. The age and gender of the individuals are shown in Supplementary Tables 2 and 3. Semiserial sections were used for OARSI grading and immunostained for SIK3 and pSIK3. OARSI grading was performed by one individual ${ }^{13}$. Use of human materials has been approved by the institutional review board of Kyoto University, University of Toyama and Hachiya Orthopaedic Hospital.

Histological analysis. For frozen sections, the samples were harvested without fixation and were immediately embedded in SCEM compound (SECTION-LAB). The sections were prepared at $6-\mu \mathrm{m}$ thickness with a Cryofilm type $2 \mathrm{c}(9)$ (SECTION-LAB) using a CM3050S cryomicrotome (Leica) according to the method described by Kawamoto ${ }^{32}$

For paraffin-embedded sections, the samples were dissected, fixed in $4 \%$ paraformaldehyde, processed and embedded in paraffin. The sections were prepared at $6-\mu \mathrm{m}$ thickness.

One individual measured the cartilage thickness and cell number of the histological sections in a blinded manner.

Introduction of osteoarthritis model into Sik $\mathbf{3}^{\mathbf{4} / \Delta}$ mice. A $100 \mu \mathrm{g}$ per gram of body weight tamoxifen (Sigma) was intraperitoneally injected into the peritoneal spaces of 7-week-old Coll 1a2-CreER; Sik $3^{\text {flox/flox }}$ and Sik flox/flox $^{\text {male mice daily }}$ for 5 consecutive days. Then, the right knee joints of the mice were subjected to DMM surgery ${ }^{33}$ or sham surgery (skin and joint capsule incision) at 8 weeks of age. The mice were killed 8 weeks after the operation, and the knee joints were subjected to histological analysis.

Rotarod performance test. The mice were put on a Rota-Rod (UGO BASILE). The speed of the rotor drums were increased from 4 to 78 r.p.m. at 0.25 r.p.m. ${ }^{-1}$ acceleration. The latency to fall from the rotor drums was recorded. The results from three trials were averaged.

Preparation of reagents. A $100 \mathrm{mg}$ tamoxifen was dissolved in $2 \mathrm{ml}$ ethanol and then suspended in $8 \mathrm{ml}$ corn oil. 4-OH tamoxifen (Sigma) was dissolved in ethano at a concentration of $0.5 \mathrm{mM}$ to prepare stock solution. Cyclophosphamide (Sigma) and bafilomycin A1 (Sigma) were dissolved in DMSO at a concentration of $100 \mathrm{mg} \mathrm{ml}^{-1}$ and $100 \mu \mathrm{M}$, respectively, to prepare stock solution. CBP-CREB interaction inhibitor (Cas 92-78-4, Merck Millipore) was also diluted in DMSO at a concentration of $20 \mathrm{mM}$ as stock solution. Recombinant mouse IL-1ß/IL-1F2 (R\&D systems) were prepared at a concentration of $10 \mu \mathrm{g} \mathrm{ml}^{-1}$ in sterile PBS with $0.1 \%$ bovine serum albumin. Ten millimolar ready to use MG132 solution (Sigma) was purchased. Pterosin B was synthesized by Intelium Corp. (Supplementary Fig. 5a). Pterosin B was dissolved in DMSO at a concentration of 100, 200 and $300 \mathrm{mM}$ for in vitro experiments and $900 \mathrm{mM}$ for mouse experiments to prepare stock solution. These solutions were diluted with culture medium for in vitro experiments.

Application of pterosin B to osteoarthritis model mice. DMM operations were performed on the right knee joints of C57BL/6NCrSlc male mice 8 weeks or 13 weeks of age. Nine hundred millimolar pterosin B stock solution was diluted with PBS to prepare the injection solution. Fifteen microlitres either of $900 \mu \mathrm{M}$ pterosin B injection solution or vehicle was injected into the intraarticular spaces of the knee joints threetimes a week. After 8 or 13 weeks of injection, mice were killed, and the knee joints were subjected to histological analysis.

OARSI scoring of articular cartilage of mouse knee joints. Twelve paraffinembedded semiserial sagittal sections with $40 \mu \mathrm{m}$ intervals were prepared from the medial compartment of each mouse knee joint. Sections were stained with safranin O-fast Green-iron haematoxylin. Cartilage destruction was scored using the OARSI scoring system ${ }^{34}$. OARSI scoring was performed by two independent scorers in a blinded manner. Six consecutive sections out of 12 were selected to represent the weight-bearing area of the femur and tibia, respectively, and were subjected to OARSI scoring. The worst three scores were selected, and their average were calculated. The scores of the femur and that of tibia were summed and designated as the OARSI score for each knee. Synovial inflammation was scored as described previously ${ }^{35}$.

Immunofluorescent staining of histological sections. Frozen sections were fixed with $4 \%$ paraformaldehyde for $5 \mathrm{~min}$. After washing with TBST, the sections were incubated with $10 \mathrm{mg} \mathrm{ml}^{-1}$ hyaluronidase (Sigma) at $37^{\circ} \mathrm{C}$ for $30 \mathrm{~min}$. After 
blocking with $10 \%$ goat normal serum (Nichirei Corporation), the sections were incubated with primary antibody for $2 \mathrm{~h}$ at room temperature.

Paraffin-embedded sections were deparaffinized and incubated in $1 \mathrm{mM}$ EDTA $(\mathrm{pH} 8.0)$ at $80^{\circ} \mathrm{C}$ for $15 \mathrm{~min}$ to retrieve the antigen. Then, sections were treated with $10 \mathrm{mg} \mathrm{ml}^{-1}$ hyaluronidase at $37^{\circ} \mathrm{C}$ for $30 \mathrm{~min}$. For detection of type $\mathrm{X}$ collagen, the samples were treated with $20 \mu \mathrm{g} \mathrm{ml}{ }^{-1}$ Proteinase K (Life Technologies) for $5 \mathrm{~min}$. After blocking with $10 \%$ goat normal serum, the sections were incubated with primary antibody for $2 \mathrm{~h}$ at room temperature.

The primary antibodies used were as follows: mouse anti-type II collagen (Thermo Scientific, 1:500), rabbit anti-type X collagen (Cosmo bio, 1:600), rabbit anti-SOX9 (Santa Cruz Biotechnology, 1:200), anti-CD31 (Abcam, 1:20) and chicken anti-GFP (for recognition of EYFP) (Abcam, 1:800). The anti-pSIK3(pT163) antibodies were raised in rabbit against peptides corresponding to residues (CSNLFTPGQLLK(pT)W, pT: phospho-Thr) of human SIK3 (Sigma, 1:2,000). Immune complexes were detected using secondary antibodies conjugated to Alexa Fluor (Life Technologies, 1:1,000). DAPI (Dojindo Molecular Technologies, 1:1,000) was used.

BrdU staining. The mice were intraperitoneally injected with BrdU labelling reagent $\left(10 \mu \mathrm{gg}^{-1}\right.$ body weight, Life Technologies) $3 \mathrm{~h}$ before being killed. The paraffin-embedded sections were prepared. The incorporated BrdU was detected using a BrdU staining kit (Life Technologies) to distinguish actively proliferating cells.

Pellet culture of mouse primary chondrocytes. Primary chondrocytes were prepared from newborn mice as described previously ${ }^{36}$. Briefly, epiphyseal cartilage was dissected from the knee joints and femoral heads of newborn mice, predigested with $3 \mathrm{mg} \mathrm{ml}^{-1}$ collagenase D (Roche) in Dulbecco's Modified Eagle Medium (DMEM) (Sigma) at $37^{\circ} \mathrm{C}$ for $90 \mathrm{~min}$ and further digested in $0.5 \mathrm{mg} \mathrm{ml}^{-1}$ collagenase D at $37^{\circ} \mathrm{C}$ overnight. A total of $5 \times 10^{5}$ primary chondrocytes were plated in a $10-\mathrm{cm}$ dish and cultured in DMEM, $10 \%$ FBS, 2 mM L-Glu (Life Technologies), $1 \%$ penicillin-streptomycin (Life Technologies) for 10 days. A total $0.5 \mu \mathrm{M} 4-\mathrm{OH}$ tamoxifen was added for the first 5 days. Then, $5 \times 10^{5}$ primary chondrocytes were transferred into a $15-\mathrm{ml}$ tube (Thermo Fisher Scientific) and centrifuged at $500 \mathrm{~g}$ for $10 \mathrm{~min}$. After 3 days incubation in the same medium in the tubes, the resulting cell pellets were transferred into petri dishes and incubated in the same medium in the presence or absence of various concentrations of pterosin B for a further 4 weeks.

Organ culture of mouse metatarsal primordial cartilage. Second, third and fourth metatarsal primordial cartilage of forelimb bud was cultured as described previously ${ }^{37}$. Metatarsal cartilage was dissected from mouse embryos at 15.5 days postcoitum. One metatarsal was organ-cultured in each well. From the next day, the metatarsals were treated with $300 \mu \mathrm{M}$ pterosin B or vehicle for 1 week. Metatarsals were subjected to histological analysis.

Pellet culture of human articular chondrocytes. Cartilage fragments were dissected from the less damaged area of the articular surface in the posterior condyle of the distal femur that was discarded at the time of total knee replacement surgery. The cartilage fragments were minced and treated with $0.25 \%$ trypsin at $37^{\circ} \mathrm{C}$ for $1 \mathrm{~h}$. Cartilage fragments were further digested in $4 \mathrm{mg} \mathrm{ml}^{-1}$ collagenase $\mathrm{D}$ at $37^{\circ} \mathrm{C}$ overnight. A total of $1 \times 10^{6}$ human articular chondrocytes were cultured in a $10-\mathrm{cm}$ dish in chondrogenic medium, which consisted of DMEM, $10 \% \mathrm{FBS}$, $1 \%$ insulin-transferrin-selenium solution (ITS, Life Technologies), $10 \mathrm{ng} \mathrm{ml}^{-1}$ recombinant human TGF- $\beta 1$ (Peprotech), $100 \mathrm{nM}$ dexamethasone (Sigma), $50 \mu \mathrm{g} \mathrm{ml}^{-1} \mathrm{~L}(+)$-ascorbic acid (Nacalai tesque), $1 \mathrm{mM}$ sodium pyruvate (Life Technologies) and $1 \%$ penicillin-streptomycin for 7 days. Then, $5 \times 10^{5}$ primary human chondrocytes were transferred into a $15-\mathrm{ml}$ tube and centrifuged at $500 \mathrm{~g}$ for $10 \mathrm{~min}$. After 3 days incubation in the same medium in the tubes, the resulting cell pellets were transferred into a petri dish and incubated in the same medium for a further 8 weeks. Finally, the medium was replaced with hypertrophic medium (DMEM supplemented with $1 \%$ FBS, $1 \%$ ITS, $100 \mathrm{ng} \mathrm{ml}^{-1}$ BMP-2 (Peprotech), $10 \mathrm{ng} \mathrm{ml}^{-1}$ GDF-5 (PTT), $1 \mu \mathrm{M}$ 3,3,5-Triiodo-L-thyronine (T3) (Sigma), $50 \mu \mathrm{g} \mathrm{ml}^{-1} \mathrm{~L}(+)$-Ascorbic acid, $10 \mathrm{nM}$ dexamethasone, $10 \mathrm{mM} \beta$-glycerophosphate (Sigma) and $1 \%$ penicillin-streptomycin) in the presence or absence of $300 \mu \mathrm{M}$ pterosin $\mathrm{B}$ for an additional 2 weeks.

\section{Hypertrophic differentiation of human iPSC-derived cartilage. Human} iPSCs (hiPSCs) were chondrogenically differentiated to produce hiPSC-derived cartilaginous particles as described previously ${ }^{38}$. The hiPSCs were transferred and then maintained in a feeder-free medium, Essential 8 (Invitrogen) with 50 units $\mathrm{ml}^{-1}$ penicillin and $50 \mathrm{mg} \mathrm{ml}$ streptomycin, in $3.5-\mathrm{cm}$ Matrigel-coated dishes. The hiPSCs formed high-density cell colonies which consisted of $1-2 \times 10^{5}$ cells 10-15 days after the start of maintenance under the feeder-free culture conditions. Subsequently, the chondrogenic differentiation of iPSCs was performed. The hiPSCs were initially differentiated into mesendodermal cells in DMEM/F12 (Sigma) with $10 \mathrm{ng} \mathrm{ml}^{-1}$ of Wnt3A (R\&D), $10 \mathrm{ng} \mathrm{ml}^{-1}$ of Activin A (R\&D), $1 \%$ ITS, $1 \%$ FBS and 50 units and $50 \mathrm{mg} \mathrm{ml}^{-1}$ of penicillin and streptomycin, respectively for 3 days. On day 3 , the medium was changed to the basal medium (DMEM with $1 \%$ ITS, $1 \% \mathrm{FBS}, 2 \mathrm{mM}$ L-glutamine (Invitrogen), $1 \times 10^{-4} \mathrm{M}$ nonessential amino acids (Invitrogen), $1 \mathrm{mM} \mathrm{Na}$ pyruvate (Invitrogen), 50 units of penicillin and $50 \mathrm{mg} \mathrm{ml}^{-1}$ of streptomycin) supplemented with $50 \mu \mathrm{g} \mathrm{ml}^{-1}$ of ascorbic acid, $10 \mathrm{ng} \mathrm{ml}^{-1}$ of BMP2, $10 \mathrm{ng} \mathrm{ml}^{-1}$ of TGF- $\beta 1$ and $10 \mathrm{ng} \mathrm{ml}^{-1}$ of GDF5, which was intended to commit the cells to the chondrocytic lineage. A total of $10 \mathrm{ng} \mathrm{ml}^{-1}$ of bFGF (WAKO) was added to the chondrogenic medium from day 3 to day 14 to increase the cell proliferation. Chondrogenic cells form multilayered nodules by day 14 . The nodules were physically separated from the bottom of the dishes to form particles, which were then transferred to a suspension culture in $3.5-\mathrm{cm}$ petri dishes on day 14 . The cells in the particles produce cartilaginous extracellular matrix, resulting in the particles becoming cartilaginous tissue in suspension culture. The culture medium was changed every 2-7 days. The hiPSC-derived cartilaginous particles on day 56 were cultured in the hypertrophic medium in the presence or absence of $300 \mu \mathrm{M}$ pterosin B for 4 weeks.

Real-time RT-PCR. The samples were frozen in liquid nitrogen and crushed by a Multibead shocker (Yasui Kikai). RNA was extracted by using ISOGEN (Nippon gene). For the real-time RT-PCR analyses, $500 \mathrm{ng}$ of total RNA was reverse-transcribed into first-strand cDNA by using ReverTra Ace (Toyobo) and random primers. PCR amplification was performed in a reaction volume of $10 \mu \mathrm{l}$ containing $1 \mu \mathrm{l} \mathrm{cDNA}$ and $5 \mu \mathrm{l}$ SYBR FAST qPCR Master Mix (Kapa Biosystems) using $7900 \mathrm{HT}$ (Life Technologies). The primers used are listed in Supplementary Tables 4 and 5 .

Sequence analysis of mRNA-seq. Primary chondrocytes of Col11a2-CreER; Sik $3^{f l o x} / f l o x$ mice were cultured in DMEM, 10\% FBS medium for 5 days on a culture dish. Then, the chondrocytes were centrifuged and incubated in tubes for 3 days. The resulting cell pellets were incubated in the same medium in the presence or absence of $300 \mu \mathrm{M}$ pterosin B for another 4 weeks. RNAs were extracted from the cultured chondrocyte pellets using QIAzol Lysis Reagent (Qiagen) and miRNeasy Mini Kit (Qiagen). RNAs were also extracted from various mouse tissues.

A 100 ng of total RNA was subjected to library preparation using KAPA stranded mRNA-seq Kits (KAPA Biosystems, Woburn, MA), according to the manufacturer's instruction. The libraries were sequenced in 100-cycle single-read mode of HiSeq2500. All sequence reads were extracted in FASTQ format using BCL2FASTQ Conversion Software 1.8.4 in the CASAVA 1.8.2 pipeline. The sequence reads were mapped to $\mathrm{mm} 9$ reference genes, using Tophat v2.0.14. Gene expression values were calculated and normalized using rpkmforgenes (10 December 2012). Averages of gene expression values from triplicate cultured chondrocyte pellet samples were calculated. Two-way hierarchical clustering of gene expressions in cultured chondrocyte pellets $(n=3)$ and tissues $(n=1)$ was conducted using the Euclidian distance and the ward method of hclust function in R3.2.1.

Gene Ontology and pathway analysis. Genes differentially expressed between control and pterosin B-treated cells were statistically selected using DEseq2 software $(|\log 2 \mathrm{FC}|>1, \mathrm{FDR}<0.05)$. GO terms over-represented in the gene list selected by DEseq2 were retrieved by hypergeometric tests using GOstats 2.36 .0 of R3.2.2 with the annotation packages GO.db 3.2.2 and org.Mm.eg.db 3.2.3. Pathway analysis was performed by hypergeometric tests of differentially expressed genes using KEGGprofile 1.12.0 of R3.2.2 with the annotation packages KEGG.db 3.2.2 and org.Mm.eg.db 3.2.3.

Cell culture. HEK293FT cells (Invitrogen) were grown in DMEM containing 10\% FBS. ATDC5 cells (Riken Cell Bank) were cultured with DMEM/Nutrient F-12 Ham (Sigma) containing 5\% FBS. GAL4-MEF2C and GAL4-CRTC2 luciferase reporter assays were performed as described previously ${ }^{39}$.

Microscope. Images were acquired on an inverted microscope (Eclipse Ti; Nikon) equipped with cameras (DS-Fi1; Nikon and C4742-80-12AG; Hamamatsu photonics), the NIS Elements software program (Nikon) and BZ-9000 (Keyence).

Immunoblot analysis. Primary chondrocytes were lysed in RIPA buffer containing protease inhibitor and phosphatase inhibitor (both Roche) and subjected to SDS-polyacrylamide gel electrophoresis. The separated proteins were then electroblotted and immunostained with rabbit anti-Sik3 antibody (1:500, Abcam), rabbit anti-phosphorylated HDAC4 (Ser246)/HDAC5 (Ser259)/HDAC7 (Ser155) antibody (Cell Signaling, 1:600), rabbit anti-HDAC4 antibody (Abcam, 1:250), rabbit anti-CRTC1 (Novus Biologicals, 1:1,000) and rabbit anti-GAPDH (Santa Cruz, 1:400). The ECL system and LAS3000 (both GE Healthcare) were used for chemiluminescent immunodetection.

Full blotting images corresponding to the immunoblottings shown in the main and Supplementary Figures are provided as Supplementary Fig. 9. 
Statistical analysis. Data are shown as averages and standard deviations. We used analysis of variance followed by the Tukey-Kramer post hoc test. In some experiments, the Student's $t$-test (two-sided) was used when homogenous variances were assumed $(P>0.05)$ by the F test, and Welch's $t$-test was used when homogenous variances were not assumed $(P<0.05)$ by the $\mathrm{F}$ test. In osteoarthritis model experiments, score values were analysed using nonparametric statistical methods (Mann-Whitney test). $P$ values $<0.05$ were considered statistically significant.

\section{References}

1. Brooks, P. M. The burden of musculoskeletal disease-a global perspective. Clin. Rheumatol. 25, 778-781 (2006).

2. Yoshimura, N. et al. Prevalence of knee osteoarthritis, lumbar spondylosis, and osteoporosis in Japanese men and women: the research on osteoarthritis/osteoporosis against disability study. J. Bone Miner. Metab. 27, 620-628 (2009).

3. Lotz, M. \& Loeser, R. F. Effects of aging on articular cartilage homeostasis. Bone 51, 241-248 (2012).

4. Hudelmaier, M. et al. Age-related changes in the morphology and deformational behavior of knee joint cartilage. Arthritis Rheum. 44, 2556-2561 (2001).

5. Echtermeyer, F. et al. Syndecan-4 regulates ADAMTS-5 activation and cartilage breakdown in osteoarthritis. Nat. Med. 15, 1072-1076 (2009).

6. Lin, A. C. et al. Modulating hedgehog signaling can attenuate the severity of osteoarthritis. Nat. Med. 15, 1421-1425 (2009).

7. Kamekura, S. et al. Osteoarthritis development in novel experimental mouse models induced by knee joint instability. Osteoarthritis Cartilage 13, 632-641 (2005).

8. Hosaka, Y. et al. Notch signaling in chondrocytes modulates endochondral ossification and osteoarthritis development. Proc. Natl Acad. Sci. USA 110, 1875-1880 (2013).

9. Saito, T. et al. Transcriptional regulation of endochondral ossification by HIF-2alpha during skeletal growth and osteoarthritis development. Nat. Med. 16, 678-686 (2010).

10. Sasagawa, S. et al. SIK3 is essential for chondrocyte hypertrophy during skeletal development in mice. Development 139, 1153-1163 (2012).

11. Berggreen, C., Henriksson, E., Jones, H. A., Morrice, N. \& Goransson, O. cAMP-elevation mediated by beta-adrenergic stimulation inhibits saltinducible kinase (SIK) 3 activity in adipocytes. Cell. Signal. 24, 1863-1871 (2012).

12. Walkinshaw, D. R. et al. The tumor suppressor kinase LKB1 activates the downstream kinases SIK2 and SIK3 to stimulate nuclear export of class IIa histone deacetylases. J. Biol. Chem. 288, 9345-9362 (2013).

13. Pritzker, K. P. et al. Osteoarthritis cartilage histopathology: grading and staging. Osteoarthritis Cartilage 14, 13-29 (2006).

14. Itoh, Y. et al. Salt-inducible kinase 3 signaling is important for the gluconeogenic programs in mouse hepatocytes. J. Biol. Chem. 290, 17879-17893 (2015)

15. Arnold, M. A. et al. MEF2C transcription factor controls chondrocyte hypertrophy and bone development. Dev. Cell 12, 377-389 (2007).

16. Lizcano, J. M. et al. LKB1 is a master kinase that activates 13 kinases of the AMPK subfamily, including MARK/PAR-1. EMBO J. 23, 833-843 (2004).

17. Sasaki, T. et al. SIK2 is a key regulator for neuronal survival after ischemia via TORC1-CREB. Neuron 69, 106-119 (2011).

18. Yamashita, A. et al. Statin treatment rescues FGFR3 skeletal dysplasia phenotypes. Nature 513, 507-511 (2014).

19. Kozhemyakina, E. et al. Identification of a Prg4-expressing articular cartilage progenitor cell population in mice. Arthritis Rheumatol. 67, 1261-1273 (2015).

20. Berdeaux, R. et al. SIK1 is a class II HDAC kinase that promotes survival of skeletal myocytes. Nat. Med. 13, 597-603 (2007).

21. Screaton, R. A. et al. The CREB coactivator TORC2 functions as a calcium- and cAMP-sensitive coincidence detector. Cell 119, 61-74 (2004).

22. Katoh, Y. et al. Salt-inducible kinase-1 represses cAMP response element-binding protein activity both in the nucleus and in the cytoplasm. Eur. J. Biochem. 271, 4307-4319 (2004).

23. Vega, R. B. et al. Histone deacetylase 4 controls chondrocyte hypertrophy during skeletogenesis. Cell 119, 555-566 (2004).

24. Cao, K. et al. Decreased histone deacetylase 4 is associated with human osteoarthritis cartilage degeneration by releasing histone deacetylase 4 inhibition of runt-related transcription factor- 2 and increasing osteoarthritis-related genes: a novel mechanism of human osteoarthritis cartilage degeneration. Arthritis Res. Ther. 16, 491 (2014).

25. Swann, D. A., Silver, F. H., Slayter, H. S., Stafford, W. \& Shore, E. The molecular structure and lubricating activity of lubricin isolated from bovine and human synovial fluids. Biochem. J. 225, 195-201 (1985).
26. Rhee, D. K. et al. The secreted glycoprotein lubricin protects cartilage surfaces and inhibits synovial cell overgrowth. J. Clin. Invest. 115, 622-631 (2005).

27. Ruan, M. Z. et al. Proteoglycan 4 expression protects against the development of osteoarthritis. Sci. Transl. Med. 5, 176ra134 (2013).

28. Ogawa, H., Kozhemyakina, E., Hung, H. H., Grodzinsky, A. J. \& Lassar, A. B. Mechanical motion promotes expression of Prg4 in articular cartilage via multiple CREB-dependent, fluid flow shear stress-induced signaling pathways. Genes Dev. 28, 127-139 (2014).

29. Uebi, T., Tamura, M., Horike, N., Hashimoto, Y. K. \& Takemori, H. Phosphorylation of the CREB-specific coactivator TORC2 at Ser(307) regulates its intracellular localization in COS-7 cells and in the mouse liver. Am. J. Physiol. Endocrinol. Metab. 299, E413-E425 (2010).

30. Yuasa, T. et al. Transient activation of Wnt/\{beta\}-catenin signaling induces abnormal growth plate closure and articular cartilage thickening in postnatal mice. Am. J. Pathol. 175, 1993-2003 (2009).

31. Tsumaki, N., Kimura, T., Matsui, Y., Nakata, K. \& Ochi, T. Separable cis-regulatory elements that contribute to tissue- and site-specific alpha 2(XI) collagen gene expression in the embryonic mouse cartilage. J. Cell Biol. 134, 1573-1582 (1996).

32. Kawamoto, T. Use of a new adhesive film for the preparation of multi-purpose fresh-frozen sections from hard tissues, whole-animals, insects and plants. Arch. Histol. Cytol. 66, 123-143 (2003).

33. Glasson, S. S., Blanchet, T. J. \& Morris, E. A. The surgical destabilization of the medial meniscus (DMM) model of osteoarthritis in the $129 / \mathrm{SvEv}$ mouse. Osteoarthritis Cartilage 15, 1061-1069 (2007).

34. Glasson, S. S., Chambers, M. G., Van Den Berg, W. B. \& Little, C. B. The OARSI histopathology initiative - recommendations for histological assessments of osteoarthritis in the mouse. Osteoarthritis Cartilage 18(Suppl 3): S17-S23 (2010).

35. Krenn, V. et al. Synovitis score: discrimination between chronic low-grade and high-grade synovitis. Histopathology 49, 358-364 (2006).

36. Gosset, M., Berenbaum, F., Thirion, S. \& Jacques, C. Primary culture and phenotyping of murine chondrocytes. Nat. Protoc. 3, 1253-1260 (2008).

37. Haaijman, A., D’Souza, R. N., Bronckers, A. L., Goei, S. W. \& Burger, E. H. OP-1 (BMP-7) affects mRNA expression of type I, II, X collagen, and matrix Gla protein in ossifying long bones in vitro. J. Bone Miner. Res. 12, 1815-1823 (1997).

38. Yamashita, A. et al. Generation of scaffoldless hyaline cartilaginous tissue from human iPSCs. Stem Cell Rep. 4, 404-418 (2015).

39. Takemori, H., Katoh Hashimoto, Y., Nakae, J., Olson, E. N. \& Okamoto, M. Inactivation of HDAC5 by SIK1 in AICAR-treated C2C12 myoblasts. Endocr. J. 56, 121-130 (2009).

\section{Acknowledgements}

We thank Yasuhiro Yamada and Akito Tanaka for the generation of Sik $3^{f l o x} /+$ mice and

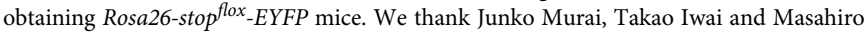
Iwamoto for the generation of Col11a2-CreER mice. We thank Hiroshi Kawaguchi and Atsushi Fukai for instruction of the mouse surgery. We also thank Peter Karagiannis for reading the manuscript and Yoshiki Minegishi, Hiromi Kishi, Takeshi Kimura and Miho Morioka for assistance and helpful discussions. We also thank Yudo Hachiya, Hiroki Watanabe (Hachiya Orthopaedic Hospital) and Ryuichi Gejo, Makiko Nogami and Mami Iwasaki (University of Toyama) for preparing the human cartilage samples, and Naoki Nishida and Koshi Kinoshita (University of Toyama) for support on the histological analysis. This study was supported in part by the Japan Science Technology Agency (JST), CREST (to N.T.), Scientific Research Grants No. 15H02561 (to N.T.) from MEXT, JST, Centers for Clinical Application Research on Specific Disease/Organ (to N.T.).

\section{Author contributions}

Y.Y. performed most of the experiments including mouse analysis and in vitro experiments. Y.Y., M.O. and A.Y. prepared $S i k 3^{f l o x} /+$ mice. H.T., Y.I., H.F. and N.K. screened pterosin B and performed the luciferase assay. Y.Y., A.K. and K.F. performed immunohistochemical and immunoblot analysis. T.K. participated in assessment of the osteoarthritis model. M.N. and A.W. performed RNA-sequencing analysis. Y.Y., N.F., H.T., T.K. and N.T. discussed the results. N.T. designed the study. Y.Y. and N.T. wrote the paper.

\section{Additional information}

Accession codes: mRNA-Seq data have been deposited in the Gene Expression Omnibus under accession code GSE75995.

Supplementary Information accompanies this paper at http://www.nature.com/ naturecommunications

Competing financial interests: The authors declare no competing financial interests. 
Reprints and permission information is available online at http://npg.nature.com/ reprintsandpermissions/

How to cite this article: Yahara, Y. et al. Pterosin B prevents chondrocyte hypertrophy and osteoarthritis in mice by inhibiting Sik3. Nat. Commun. 7:10959 doi: $10.1038 /$ ncomms10959 (2016). (c) (i) This work is licensed under a Creative Commons Attribution 4.0 International License. The images or other third party material in this article are included in the article's Creative Commons license, unless indicated otherwise in the credit line; if the material is not included under the Creative Commons license, users will need to obtain permission from the license holder to reproduce the material. To view a copy of this license, visit http://creativecommons.org/licenses/by/4.0/ 


\section{Corrigendum: Pterosin B prevents chondrocyte} hypertrophy and osteoarthritis in mice by inhibiting Sik3

Yasuhito Yahara, Hiroshi Takemori, Minoru Okada, Azuma Kosai, Akihiro Yamashita, Tomohito Kobayashi, Kaori Fujita, Yumi Itoh, Masahiro Nakamura, Hiroyuki Fuchino, Nobuo Kawahara, Naoshi Fukui, Akira Watanabe, Tomoatsu Kimura \& Noriyuki Tsumaki

Nature Communications 7:10959 doi: 10.1038/ncomms10959 (2016); Published 24 Mar 2016; Updated 27 Jun 2016

In this Article, there is an error in the labelling of the western blot in Fig. 3f. The labels indicating the presence of pterosin B in each lane should read ' -+++ '. The correct version of Fig. 3 appears below. 
a<smiles>Cc1cc2c(c(C)c1CCO)C(=O)C(C)C2</smiles>

Pterosin B b

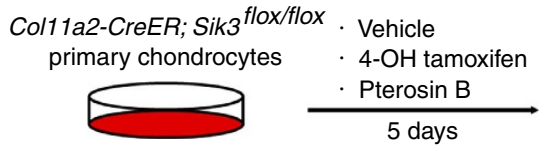

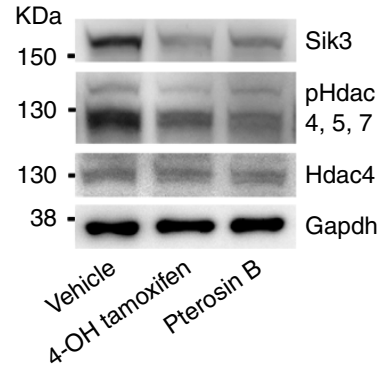

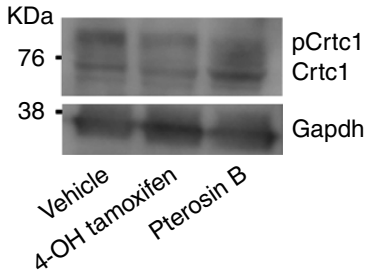

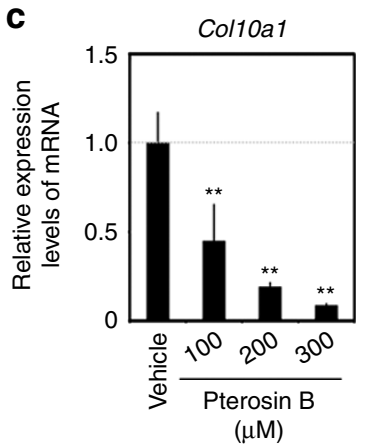
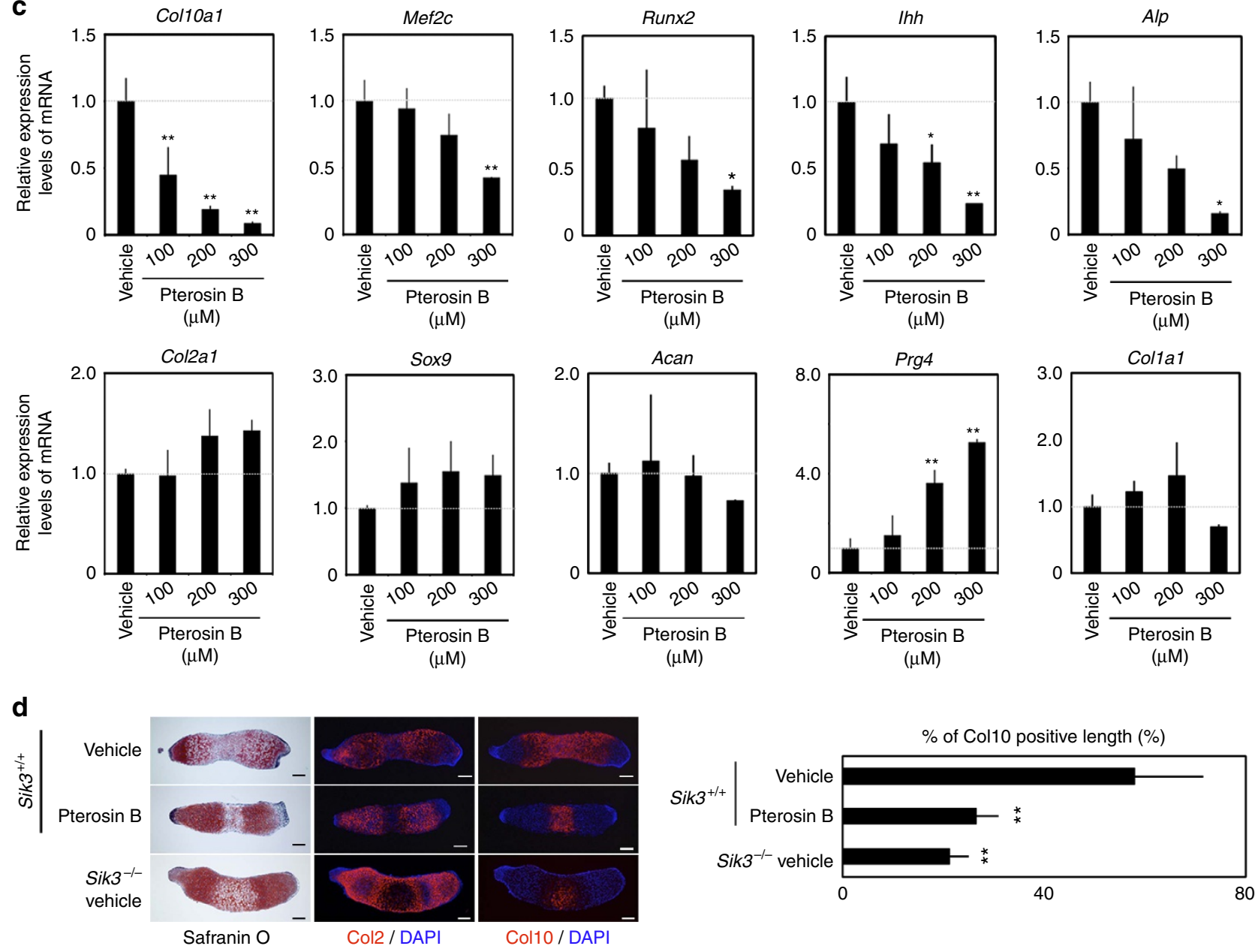

e

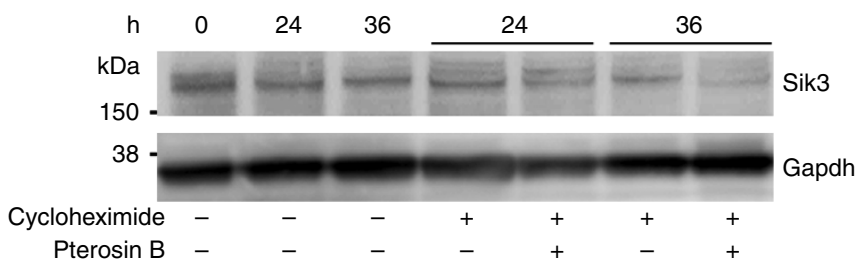

f

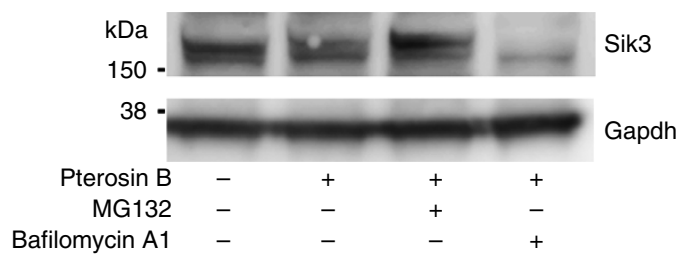

Figure 3

(c) This work is licensed under a Creative Commons Attribution 4.0 International License. The images or other third party material in this article are included in the article's Creative Commons license, unless indicated otherwise in the credit line; if the material is not included under the Creative Commons license, users will need to obtain permission from the license holder to reproduce the material. To view a copy of this license, visit http://creativecommons.org/licenses/by/4.0/ 